\title{
Analysis of CMOS Compatible Cu-Based TM-Pass Optical Polarizer
}

\author{
Tien Khee Ng, Member, IEEE, Mohammed Zahed Mustafa Khan, Ahmad Al-Jabr, \\ and Boon S. Ooi, Senior Member, IEEE
}

\begin{abstract}
A transverse-magnetic-pass (TM-pass) optical polarizer based on $\mathrm{Cu}$ complementary metal-oxidesemiconductor technology platform is proposed and analyzed using the 2-D method-of-lines numerical model. In designing the optimum configuration for the polarizer, it was found that the metal-insulator-metal (MIM) polarizer structure is superior compared to the insulator-metal-insulator polarizer structure due to its higher polarization extinction ratio $(P E R)$ and low insertion loss. An optimized MIM TM-pass polarizer exhibits simulated long wavelength pass filter characteristics of $>\sim 1.2 \mu \mathrm{m}$, with fundamental $\mathrm{TM}_{0}$ and $\mathrm{TE}_{0}$ mode transmissivity of $>70 \%$ and $<5 \%$, respectively, and with $P E R \sim 11.5 \mathrm{~dB}$ in the wavelength range of 1.2-1.6 $\mu \mathrm{m}$. The subwavelength and submicrometer features of this TM-polarizer are potentially suitable for compact and low power photonics integrated circuit implementation on silicon-based substrates.
\end{abstract}

Index Terms-Complementary metal-oxide-semiconductor compatible, integrated photonics, numerical method, optical polarizer, silicon photonics.

\section{INTRODUCTION}

A RECENT advancement in the active optical components research involves the use of broad gain spectrum material structure, such as as-grown and intermixed quantum-dots (QD) [1] and as-grown quantum-dash (Qdash) [2]-[4] material structure, where a broad electroluminescent linewidth were achieved for such emitters, in contrast to short linewidth QDs structures [5]-[7]. The availability of the broad gain spectrum devices is significant for broad wavelength tunability applications, such as in optical communications [8], [9], imaging [10], metrology, spectroscopy and sensing [11].

The motivation of this work stems from the potential need to process wavelength information detected from the transmission or reflection of such broad-spectrum. Also, new optical components are required for implementing various optical functions in photonics integrated circuits (PICs) with a small footprint. The broad-wavelength emission is particularly suited for compact optical coherent tomography (OCT) [10], [12]. Specifically, the transverse magnetic (TM) mode field

Manuscript received Oct 29, 2011; revised January 31, 2012; accepted February 2, 2012. Date of publication February 10, 2012; date of current version April 4, 2012. This work was supported in part by KAUST - University of Michigan Academic Excellence Alliance Grant 2010.

The authors are with the Photonics Laboratory, King Abdullah University of Science and Technology, Thuwal 23955-6900, Saudi Arabia (e-mail: tienkhee.ng@kaust.edu.sa; mohammedzahed.khan@kaust.edu.sa; ahmad.aljabr@kaust.edu.sa; bsooi@ieee.org).

Color versions of one or more of the figures in this letter are available online at http://ieeexplore.ieee.org.

Digital Object Identifier 10.1109/LPT.2012.2187329 is preferred for such application compared to the transverse electric (TE) mode field [13] due to its polarization sensitivity. Furthermore, having a combination of phase information derived from the TE and TM signal is potentially desirable for near infra-red (NIR) polarization-sensitive OCT [14] design for scanning of human tissues exhibiting birefringence properties. Hence, we propose the design of a $\mathrm{Cu}$-based TM-pass polarizer with long-pass characteristic suitable for processing the O-, E-, S-, C-, L-, U-band (OESCLU-band) wavelength information, while suppressing the short wavelength light (UV-visible-NIR). Two Cu-based polarizer designs, the IMI and MIM schemes, were investigated using 2D MoL model.

\section{Design and Simulation Details}

Consider the 2D time harmonic wave equation given by

$$
\frac{\partial^{2} \psi}{\partial x^{2}}+\frac{\partial^{2} \psi}{\partial z^{2}}+k_{0}^{2} n^{2} \psi=0
$$

where $\psi=\psi(x, z)$ represents the electric field $E_{y}$ of $\mathrm{TE}$ polarized waves or magnetic field, $H_{y}$ for TM polarized wave. $k_{0}=2 \pi / \lambda$ is the free space wave number, and $\underline{n}=n(x)$ is the refractive index. The $\mathrm{Cu}$ optical constants [15] change with the operating wavelength, and the refractive index of $\mathrm{SiO}_{2}$ and $\mathrm{Si}$ are 1.5 and 3.5 , respectively. Upon discretization of $\partial^{2} \psi / \partial x^{2}$ into $N$ sample points of mesh size $\Delta x$, using the central difference approximation, Eq. 1 has a general solution given by [16]-[18]:

$$
\bar{\Psi}(z)=e^{j \bar{S} z} A+e^{-j \bar{S} z} B
$$

where the column vector $\bar{\Psi}(z)$ contains the discretized electric field or magnetic field. The square matrix $\bar{S}$ is given by $\bar{S}^{2}=\bar{D}_{x x}^{2}+k_{0}^{2} \bar{N}^{2}$, where the matrix $\bar{D}_{x x}^{2}$ is the transverse second-order derivative operator, and $\bar{N}^{2}$ is the square of $n$. Simulations are performed considering the $2 \mathrm{D}$ structure in the $x-z$ plane, and encompassing a perfectly matched absorbing layer on either side of the computational window to absorb the radiative field. The polarizer is launched with the fundamental mode of the input waveguide at $1.55 \mu \mathrm{m}$ from the left (at the input waveguide - polarizer junction). The $\mathrm{TM}_{0}$ and $\mathrm{TE}_{0}$ mode transmissivity $\left(P_{T M 0}\right.$ and $P_{T E 0}$, respectively) defined as the ratio of the transmitted mode power to the incident mode power, is calculated using the overlap integral, and at the polarizer - output waveguide junction. The $P E R$, and $\mathrm{TM}_{0}$ insertion loss $(I L)$ are $-10 \log _{10}\left(P_{T M 0} / P_{T E 0}\right)$, and $-10 \log _{10}\left(P_{T M 0}\right)$, respectively. 


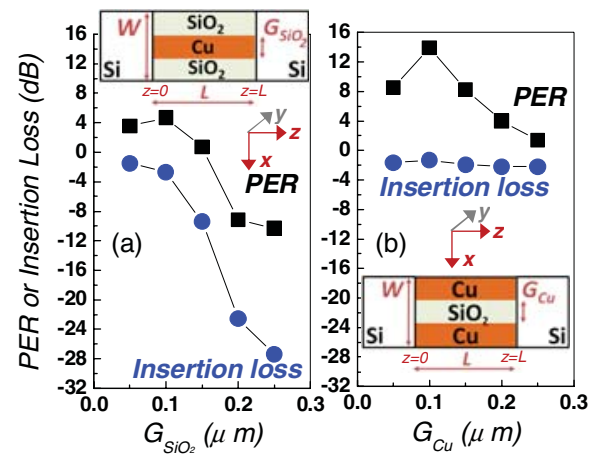

Fig. 1. PER or $I L$ versus (a) gap between $\mathrm{SiO}_{2}$ strips $\left(G_{\mathrm{SiO}_{2}}\right)$ and (b) gap between $\mathrm{Cu}$ strips $\left(G_{\mathrm{Cu}}\right)$ for IMI and MIM structures, respectively. Insets show the $x-z$ plane of the structures with $L=0.1 \mu \mathrm{m}$ and $W=0.3 \mu \mathrm{m}$.

The $\mathrm{SiO}_{2}$ encapsulated device structure consists of a $\mathrm{Si}$ core/Cu-based polarizer/Si-core along the longitudinal or $z$ direction (direction of wave propagation) with a fixed width $(W)$ of $0.3 \mu \mathrm{m}$ in the transverse or $x$ direction. The Si-cores at both ends of the structure constitute the input and output waveguides segments, while the middle segment functions as a polarizer constructed using $\mathrm{Cu}$ and $\mathrm{SiO}_{2}$. The width of the $\mathrm{Si}$-core is selected to ensure single-mode, broad-wavelength operation. It is also noted that $\mathrm{Si}$ channel waveguides used in complementary metal-oxide-semiconductor (CMOS) compatible photonics are polarization sensitive.

\section{RESULTS AND DISCUSSION}

The $x-z$ plane of the two polarizer configurations, IMI and MIM, selected for the analysis is shown in the insets of Fig. 1. The IMI TM-polarizer configuration has a fixed polarizer length, $L$, and width, $W$, of 0.1 and $0.3 \mu \mathrm{m}$, respectively. In the simulation, the $\mathrm{Cu}$ filled $\mathrm{SiO}_{2}$ gap, $G_{\mathrm{SiO} O}$, was increased from $0.05 \mu \mathrm{m}$ to $0.25 \mu \mathrm{m}$ along the transverse direction. In Fig. 1(a), the PER values of less than $4.7 \mathrm{~dB}$ are obtained for $G_{S i O 2}<\sim 0.15 \mu \mathrm{m}$ (see Fig. 1(a)). For $G_{S i O 2}>0.15 \mu \mathrm{m}$, negative $P E R$ values of up to $-10 \mathrm{~dB}$ were obtained. This is because of the increase in the losses of the $\mathrm{TM}_{0}$ evanescent wave generated at the $\mathrm{Cu}-\mathrm{SiO}_{2}$ interface causing a decrease in $\mathrm{TM}_{0}$ mode transmissivity. This is evident in the corresponding $I L$, which decreases significantly from $\sim-10 \mathrm{~dB}$ at $G_{\mathrm{SiO} 2}=$ $0.15 \mu \mathrm{m}$ towards $-30 \mathrm{~dB}$ at $G_{S i O 2}=0.25 \mu \mathrm{m}$. Such a scheme is hence undesirable.

A TM evanescent wave confinement and guiding scheme is obviously required. More importantly, the scheme should ideally propagate the TM wave and suppress the TE wave. This calls for the MIM scheme as the most obvious candidate as TM wave can be supported by the metal-insulator interface, and metal-sandwich structure at sub-wavelength features size and would block much of the unsupported TE mode. The simulation results of this scheme are presented in Fig. 1(b). As evident from the figure, the low $I L$ values of between $-0.5 \mathrm{~dB}$ and $-2 \mathrm{~dB}$ were maintained for the MIM scheme as the $\mathrm{SiO}_{2}$ filled $\mathrm{Cu}$ gap, $G_{C u}$, was increased from $0.05 \mu \mathrm{m}$ to $0.25 \mu \mathrm{m}$. A negligible increase in the $I L$ with $L$ is expected from the energy dissipation from metal absorption. Positive PER values indicated a configuration that favors TM transmission
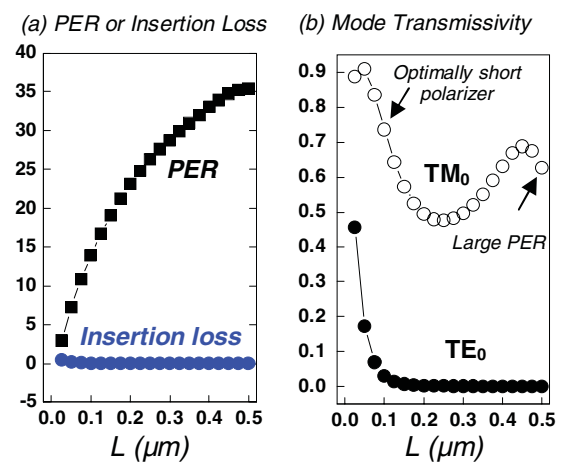

Fig. 2. Plot of (a) PER or $\mathrm{TM}_{0}$ insertion loss (IL) and (b) fundamental modes transmissivity versus $L$ with $G_{\mathrm{Cu}}=0.1 \mu \mathrm{m}$ and $W=0.3 \mu \mathrm{m}$.

and with a maximum value of $+13.9 \mathrm{~dB}$ at $0.1 \mu \mathrm{m} G_{C u}$. Keeping the optimum $G_{C u}$ at $0.1 \mu \mathrm{m}$, and $W=0.3 \mu \mathrm{m}$, the MIM scheme showed a large PER of up to $\sim 35.4 \mathrm{~dB}$ and $I L$ of less than $\sim-3.2 \mathrm{~dB}$ throughout, as $L$ was increased to $0.5 \mu \mathrm{m}$, as shown in Fig. 2(a). The corresponding $\mathrm{TM}_{0}$ and $\mathrm{TE}_{0}$ mode transmissivity for calculations of $P E R$ are shown on Fig. 2(b). The large PER of $35.4 \mathrm{~dB}$ corresponds to a $\mathrm{TM}_{0}$ mode transmissivity of $\sim 0.6$ and reflectivity of $<0.05$, with $L=0.5 \mu \mathrm{m}$, while an optimally short polarizer $(L=0.1 \mu \mathrm{m})$ having moderate $P E R$ of 14 but with a higher $\mathrm{TM}_{0}$ mode transmissivity of $>0.7$ and reflectivity of $<0.013$ can also be achieved. The respective fabrication tolerances of $+/-0.1 \mathrm{~dB}$ and $+/-1 \mathrm{~dB}$ can be achieved given the worst case $L$ variation of $+/-10 \%$ or $+/-10 \mathrm{~nm}$. This gives flexibility in design and fabrication.

To investigate the wavelength transmission characteristics, the MIM polarizer was launched with the fundamental mode of the input wavelength between 0.4 and $1.6 \mu \mathrm{m}$, and the corresponding complex refractive indexes or optical constants $(n-k)$ for $\mathrm{Cu}$ at the corresponding wavelengths were used to compute the $\mathrm{TM}_{0}$ and $\mathrm{TE}_{0}$ mode transmissivity. Fig. 3(a) shows that the polarizer exhibited a simulated long wavelength pass filter characteristics, with $\mathrm{TM}_{0}$ and $\mathrm{TE}_{0}$ mode transmissivity of $>70 \%$ and $<5 \%$, respectively, at $1.2-1.6 \mu \mathrm{m}$ wavelength range with $P E R$ of $\sim+11.5 \mathrm{~dB}$. The long pass transmission beyond $1.2 \mu \mathrm{m}$ is a property of the polarizer unit exclusively since the numerical model does not consider the $\mathrm{Si}$ waveguide absorption in the numerical model (the theoretical formulation). The simulated magnetic and electric field intensity images for the $\mathrm{TM}_{0}$ and $\mathrm{TE}_{0}$ modes at $1.55 \mu \mathrm{m}$ wavelength are shown in Fig. 3(b) and 3(c), respectively, where the $\mathrm{TM}_{0}$-pass and $\mathrm{TE}_{0}$-reject characteristics are clearly observed. The standing wave in Fig. 3(c) is due to wave interference.

The selective mode segregation is due to the surfaceplasmon-polaritons (SPPs) phenomenon. SPPs are TM electromagnetic waves that propagate at the interface between two media with opposite sign of permittivity $\left(\varepsilon=n^{2}\right)$, such as metal and dielectrics. The surfaces of metals have very high concentration of free electrons, and exhibit negative permittivity response when illuminated [19]. In our case, $\mathrm{Cu}$ and $\mathrm{SiO}_{2}$ in the MIM structure form a cavity with dimensions below the diffraction limit. Light cannot propagate unless it is in the form of SPPs excited by TM mode [20]. The input $\mathrm{TM}_{0}$ mode 

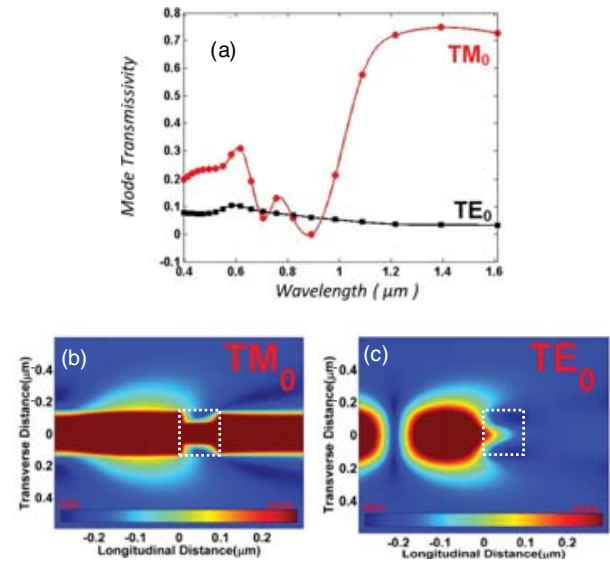

Fig. 3. (a) Fundamental modes transmissivity versus wavelength, (b) magnetic field intensity image of the $\mathrm{TM}_{0}$ mode, and (c) electric field intensity image of the $\mathrm{TE}_{0}$ mode, at $1.55 \mu \mathrm{m} . G_{\mathrm{Cu}}=L=0.1 \mu \mathrm{m}, W=$ $0.3 \mu \mathrm{m}$, and the polarizer (dashed box)/input waveguide junction is located at $(z, x)=(0,0)$.

power hence propagates through the $\mathrm{Cu}-\mathrm{SiO}_{2}$ interfaces in the proposed polarizer while the $\mathrm{TE}_{0}$ mode power is blocked.

This TM-polarizer configuration competes favorably with polarizers based on corrugated waveguide, photonics crystal waveguide [21], slotted waveguide [22], and sandwiched waveguide [23] structures. The major advantage of our proposed waveguide structure in segregating the $\mathrm{TM}_{0}$ and $\mathrm{TE}_{0}$ modes is evident from the sub-micron feature sizes, despite its high $\mathrm{TM}_{0}$ reflection and radiation loss of $<0.30$ $(<0.05$ and $<0.25$, respectively) for $1.2-1.6 \mu \mathrm{m}$ wavelength range. The proposed TM-pass photonic element is practical for high density PICs based on SOI and $\mathrm{Cu}$-Damascene CMOS foundry platforms [24], [25].

Although this study focuses on the passive component implementation, the fundamental building block can also be modified for electro-optical modulation by extending the $\mathrm{Cu}$ metal layers for external biasing of an embedded semiconductor. The feasibility of integrating a small foot-print structure to realize various optical functions, such as a long-wavelength pass filter, a TM-polarizer and potentially an electro-optical modulator with CMOS process compatible features further elucidate the significance of this work.

\section{CONCLUSiON}

In summary, we proposed here a CMOS compatible long wavelength pass $\mathrm{TM}_{0}$-polarizer. In an optimally designed MIM polarizer, the $\mathrm{TM}_{0}$ and $\mathrm{TE}_{0}$ mode transmissivity of $>70 \%$ and $<5 \%$, respectively, in the wavelength range of $1.2-1.6 \mu \mathrm{m}$, can be achieved with $G_{C u}=L=0.1 \mu \mathrm{m}$ and $W=0.5 \mu \mathrm{m}$. The proposed structure has a small foot-print with $P E R \sim$ $+11.5 \mathrm{~dB}$ when implemented as a $\mathrm{TM}_{0}$-polarizer across the long-pass wavelength regime.

\section{REFERENCES}

[1] H. S. Djie, et al., "Ultrabroad stimulated emission from quantumdash laser," Appl. Phys. Lett., vol. 91, no. 11, pp. 111116-1-111116-3, Sep. 2007

[2] B. S. Ooi, et al., "Quantum dashes on InP substrate for broadband emitter applications," IEEE J. Sel. Topics Quantum Electron., vol. 14, no. 4, pp. 1230-1238, Jul./Aug. 2008.
[3] M. Z. M. Khan, T. K. Ng, U. Schwingenschlogl, and B. S. Ooi, "Modeling the lasing spectra of InAs/InP Quantum dash lasers," Appl. Phys. Lett., vol. 98, no. 10, pp. 101105-1-101105-3, Mar. 2011.

[4] M. Z. M. Khan, T. K. Ng, U. Schwingenschlogl, and B. S. Ooi, "Effect of the number of stacking layers on the characteristics of quantumdash lasers," Opt. Express, vol. 19, no. 14, pp. 13378-13385, 2011.

[5] D. Bordel, et al., "Growth of InAs/GaAs quantum dots on germaniumon-insulator-on-silicon (GeOI) substrate with high optical quality at room temperature in the $1.3 \mu \mathrm{m}$ band," Appl. Phys. Lett., vol. 96, no. 4, pp. 043101-1-043101-3, 2010.

[6] D. Bimberg, N. Kirstaedter, N. N. Ledentsov, Z. I. Alferov, P. S Kop'ev, and V. M. Ustinov, "InGaAs-GaAs quantum-dot lasers," IEEE J. Sel. Topics Quantum Electron., vol. 3, no. 2, pp. 196-205, Apr. 1997.

[7] Z. Y. Zhang, R. A. Hogg, X. Q. Lv, and Z. G. Wang, "Self-assembled quantum-dot superluminescent light-emitting diodes," Adv. Opt. Photon., vol. 2, no. 2, pp. 201-228, Jun. 2010.

[8] B. C. Collings, M. L. Mitchell, L. Boivin, and W. H. Knox, "A 1021 channel WDM system," IEEE Photon. Technol. Lett., vol. 12, no. 7, pp. 906-908, Jul. 2000.

[9] A. Akrout, et al., "Separate error-free transmission of eight channels at $10 \mathrm{~Gb} / \mathrm{s}$ using comb generation in a quantum-dash-based mode-locked laser," IEEE Photon. Technol. Lett., vol. 21, no. 23, pp. 1746-1748, Dec. 1, 2009.

[10] D. Huang, et al., "Optical coherence tomography," Science, vol. 254, no. 5035, pp. 1178-1181, 1991.

[11] H. I. Schiff, G. I. Mackay, and J. Bechara, "The use of tunable diode laser absorption spectroscopy for atmospheric measurements," in Air Monitoring by Spectroscopic Techniques, M. W. Sigrist, Ed. New York: Wiley, 1994, pp. 239-344.

[12] C. E. Dimas, C. L. Tan, H. S. Djie, A. D. McAulay, and B. S. Ooi, "A temporal coherence study of quantum-dot/dash broadband lasers and superluminescent diodes," IEEE Photon. Technol. Lett., vol. 21, no. 11, pp. 694-696, Jun. 1, 2009.

[13] A. Densmore, et al., "A silicon-on-insulator photonic wire based evanescent field sensor," IEEE Photon. Technol. Lett., vol. 18, no. 23, pp. 2520-2522, Dec. 1, 2006.

[14] J. M. Schmitt, "Optical coherence tomography (OCT): A review," IEEE J. Sel. Topics Quantum Electron., vol. 5, no. 4, pp. 1205-1215, Jul./Aug. 1999.

[15] P. B. Johnson and R. W. Christy, "Optical constants of the noble metals," Phys. Rev. B, vol. 6, no. 12, pp. 4370-4379, 1972.

[16] H. A. Jamid, M. Z. M. Khan, and M. Ameeruddin, "A compact $90^{\circ}$ threebranch beam splitter based on resonant coupling," J. Lightw. Technol., vol. 23, no. 11, pp. 3900-3906, Nov. 2005.

[17] M. Z. M. Khan and H. A. Jamid, "Full-vectorial analysis of highindexcontrast coupled channel waveguides," Appl. Opt., vol. 48, no. 22, pp. 4468-4474, 2009.

[18] H. A. Jamid and M. Z. M. Khan, "A noniterative formulation for 2-D optical waveguide discontinuity problems based on Padé approximants," IEEE Photon. Technol. Lett., vol. 20, no. 7, pp. 469-471, Apr. 1, 2008.

[19] S. A. Maier, M. L. Brongersma, P. G. Kik, S. Meltzer, A. A. G. Requicha, and H. A. Atwater, "Plasmonics: A route to nanoscale optical devices," Adv. Mater, vol. 13, no. 19, pp. 1-2, 2001.

[20] S. A. Maier, Plasmonics: Fundamentals and Applications. Berlin, Germany: Springer-Verlag, 2007.

[21] Y. Cui, W. Qi, E. Schonbrun, M. Tinker, J. B. Lee, and W. Park, "Siliconbased 2-D slab photonic crystal TM polarizer at telecommunication wavelength," IEEE Photon. Technol. Lett., vol. 20, no. 8, pp. 641-643, Apr. 15, 2008.

[22] C.-H. Chen, L. Pang, C.-H. Tsai, U. Levy, and Y. Fainman, "Compact and integrated TM-pass waveguide polarizer," Opt. Express, vol. 13, no. 14, pp. 5347-5352, 2005.

[23] W. Qian and S. T. Ho, "Ultracompact TM-pass silicon nanophotonic waveguide polarizer and design," IEEE Photon. J., vol. 2, no. 1, pp. 49-56, Feb. 2010.

[24] S. Lakshminarayanan, et al., "Contact and via structures with copper interconnects fabricated using dual Damascene technology," IEEE Electron Device Lett., vol. 15, no. 8, pp. 307-309, Aug. 1994.

[25] P. Dumon, et al., "Linear and nonlinear nanophotonic devices based on silicon-on-insulator wire waveguides," Jpn. J. Appl. Phys., vol. 45, no. 8B, pp. 6589-6602, 2006. 